\title{
Acute infantile liver failure-multisystemic involvement syndrome
}

INSERM

\section{Source}

INSERM. (1999). Orphanet: an online rare disease and orphan drug data base. Acute infantile liver failure-multisystemic involvement syndrome. ORPHA:370088

Acute infantile liver failure-multisystemic involvement syndrome is a rare, genetic, parenchymal hepatic disease characterized by acute liver failure, that occurs in the first year of life, which manifests with failure to thrive, hypotonia, moderate global developmental delay, seizures, abnormal liver function tests, microcytic anemia and elevated serum lactate. Other associated features include hepatosteatosis and fibrosis, abnormal brain morphology, and renal tubulopathy. Minor illness exacerbates deterioration of liver failure. 